\title{
Original Article \\ An Isolate of Arthroderma benhamiae with Trichophyton mentagrophytes var. erinacei Anamorph Isolated from a Four-Toed Hedgehog (Atelerix albiventris) in Japan
}

\author{
Yoko Takahashi ${ }^{1}$, Kumiko Haritani ${ }^{2}$, Ayako Sano ${ }^{1}$, Kayoko Takizawa ${ }^{1}$, \\ Kazutaka Fukushima ${ }^{1}$, Makoto Miyaji ${ }^{1}$, Kazuko Nishimura ${ }^{1}$ \\ 'Research Center for Pathogenic Fungi and Microbial Toxicoses, Chiba University, \\ 1-8-1 Inohana, Chuo-ku, Chiba 260-8673, Japan \\ 2 Namiki Animal Hospital, 7-2-4 Namiki Abiko, Chiba 270-1165, Japan
}

[Received: 14, December 2001, Accepted: 1, July 2002]

\begin{abstract}
A female four-toed hedgehog probably imported from Africa and kept as a pet by a family suffered from depilation and mite (Caparinia tripilis) infection. Depilated quills were inoculated on a commercially available medium and an isolate of the dermatophytes was obtained. A giant colony after 14 days incubation on yeast extract Sabouraud's agar had a central umbo with white granular surface and a yellow pigment ring in the reverse. The hedgehog isolate produced numerous elongated microconidia singly attached along the sides of hyphae. Macroconidia were somewhat irregular in shape and size and 2-6 septa. Abundant intermediate sized spores between micro- and macro conidia and few spirals were observed. Hair perforation and urease activity tests were positive. Maximum growth temperature was $40^{\circ} \mathrm{C}$. In the mating tests using the tester strains of both African and Americano-European races of Arthroderma benhamiae, the strain produced numerous gymnothecia only when paired with the African race mating type minus (-). In addition, 591 bases of the internal transcribed spacer region of the ribosomal RNA gene including the 5.8S region (ITS1-5.8S-ITS2) were sequenced and corresponded to those of $T$. mentagrophytes var. erinacei (DDBJ/EMBL/GenBank accession numbers Z97996 and Z97997) by more than 99.7\%. Therefore, our case is the first isolation of $A$. benhamiae with $T$. mentagrophytes var. erinacei anamorph in Japan.
\end{abstract}

Key words: Arthroderma benhamiae, Atelerix albiventris, hedgehog, Trichophyton mentagrophytes var. erinacei

\section{Introduction}

Owing to the recent pet boom, the species of animals being kept in the home has diversified, and a succession of rare species have been imported from foreign countries. Reports of dermatophytes isolated from these animals have also increased ${ }^{1)}$. Rodents such as hamsters ${ }^{2}$, rabbits $^{3)}$, rats $^{4)}$, and chinchillas ${ }^{2)}$ are often reported with Trichophyton mentagrophytes, as well as dogs and cats. The current principal causative organism is Arthroderma vanbreuseghemii, followed by $A$. benhamiae ${ }^{5,6)}$ which was first reported in

Address for correspondence: Kazuko Nishimura

Research Center for Pathogenic Fungi and Microbial

Toxicoses, Chiba University

1-8-1 Inohana Chuo-ku, Chiba 260-8673, Japan
Japan only a few years ago.

The present study introduces the isolation and identification of a dermatophyte from tinea in a four-toed hedgehog (Atelerix albiventris $)^{7)}$, family Erinaceidae, order Insectivora. The animal strain was investigated by morphological, molecular biological and mating studies, and identified as $A$. benhamiae, mating type plus $(+)$ in teleomorph and as $T$. mentagrophytes var. erinacei in anamorph.

Until this report, there had been no case of $T$. mentagrophytes var. erinacei infection in Japan, and this is the first isolation of the fungus from a household hedgehog. As imports of various pet animals increase, pathogenic fungi which have not previously existed in our country will be isolated and new problems are likely to follow. 


\section{Case}

Animal: A female four-toed hedgehog (Atelerix albiventris (Wagner, 1841; Corbet, 1988)), presumed to be about 2 years of age and weighing about $400 \mathrm{~g}$ was investigated. She was purchased at a pet shop about two years ago. According to the owner who lives in Abiko, Chiba Prefecture, Japan, the animal's natural habitat was Africa, but details of how it was brought to Japan were unknown. The owners were a man and his wife in their 20s; they had no past history of dermatophytosis, although the wife was receiving treatment for atopic dermatitis. The hedgehog had been kept in a separate indoor cage as had one male and one other female four-toed hedgehog and one barking squirrel which had been purchased later. The other animals were clinically healthy. The first time the animal was brought to the clinic was August 9, 2001.

Past history: Nothing to be noted.

Present illness: When the animal was first obtained, the owners noticed dry skin and massive scales on it, but did nothing for the symptoms. The day before the first visit to the clinic, they found small white, mite-like deposits on the body surface. The hedgehog had often scratched her body, and she was brought to the clinic so that parasitological and mycological examinations could be made.

Present symptoms: On the first visit, the quills were somewhat sparse from the area of the dorsal site to the lateral region, and the skin under the quills was covered with scales and a crust. When touched, the quills dropped easily, as though breaking off from their roots. The animal showed a severe depilation of quills when her body trembled. Figure 1 shows the skin after quills had dropped; parasitism by many mites was seen, and the $\mathrm{KOH}$ specimen of the dorsal crust revealed many Caparinia tripilis mites.

Mycological findings: The dorsal quills were cultured on modified Sabouraud's agar supplemented with antibiotics and a $\mathrm{pH}$ indicator (TME; Nikken-Seibutsu-Igaku-Kenkyujyo, Kyoto, Japan) at room temperature, and formed a whitish powdery colony centered in the hair root. A giant colony cultured on a yeast extract added Sabouraud's agar medium (glucose $2 \%$, peptone $1 \%$, yeast extract $0.5 \%$, agar $1.5 \%$ ) at $25^{\circ} \mathrm{C}$ was morphologically examined. On the 14 th day, the obverse of the colony showed a cottony elevation in the center, and a white, powdery and flattened texture in the surrounding area (Fig. 2a). On the reverse, the center was yellowish brown, and orange-yellow pigment diffused in a ring shape just inside the surrounding area (Fig. 2b). On

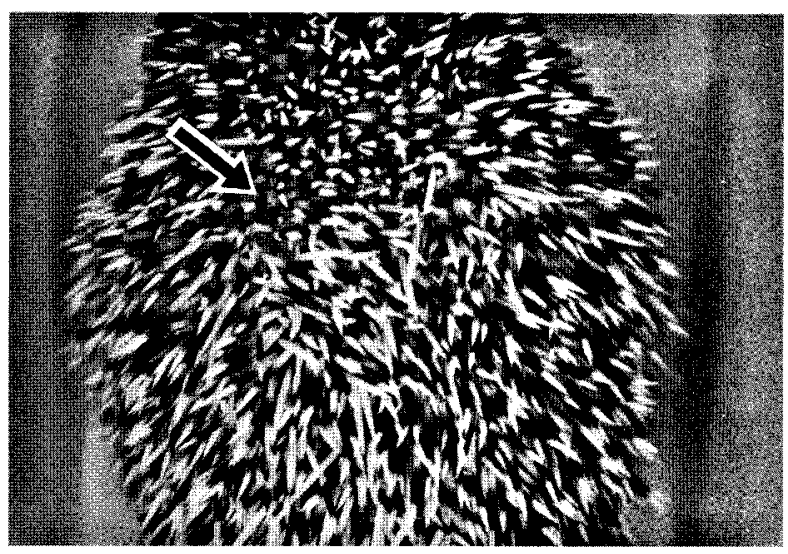

Fig. 1 Four-toed hedgehog (Atelerix albiventris) infected with $T$ mentagrophytes var. erinacei on the back (arrow).

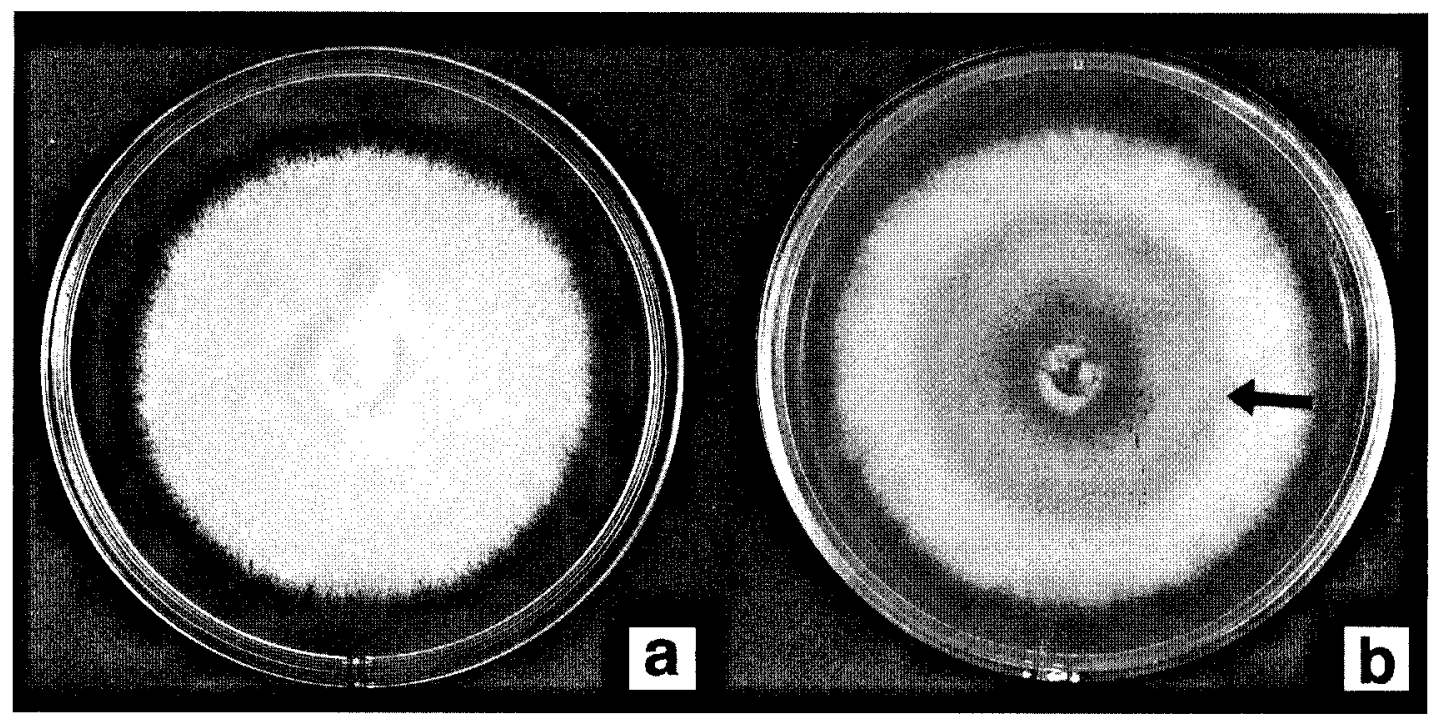

Fig. 2 Culture on YSB at $25^{\circ} \mathrm{C}$ for 2 weeks.

a: Obverse, finely granular with central downy umbo.

b: Reverse, orange yellow pigment developed as a ring (arrow). 


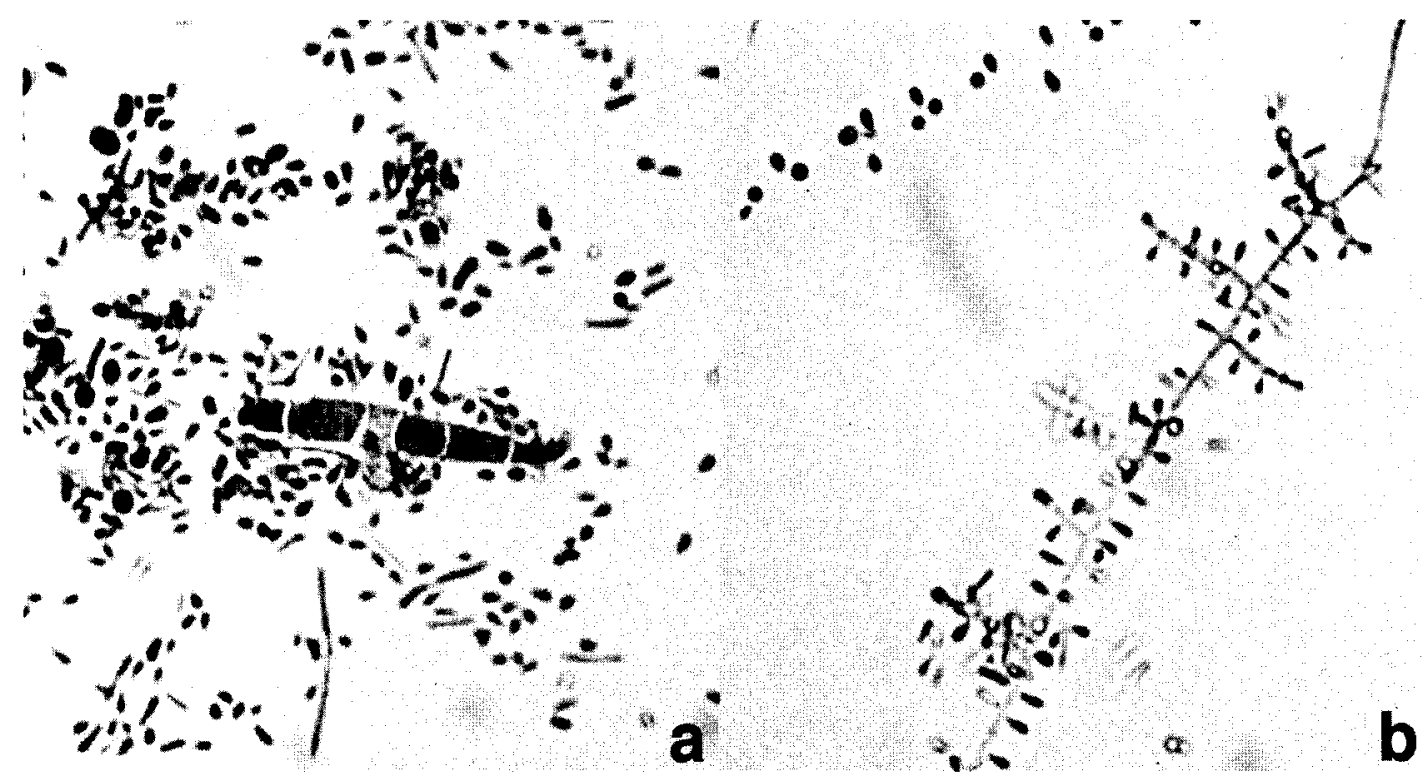

Fig. 3 Micromorphology in PDA slide culture (Lactophenol cotton blue staining, $\times 400$ ).

a: Irregular shaped and sized macroconidia with 2-6 septa.

b: Pear- or more elongated shaped microconidia attached at right-angles along the sides of hyphae.

potato dextrose agar plates (PDA: Difco, Detroit, MI, USA), the colony had lemon-yellow pigment diffused in a thin ring shape on the reverse. However, the pigment produced in neither medium was bright.

In a slide culture using PDA $\left(25^{\circ} \mathrm{C}, 14\right.$ days $)$, a few macroconidia were observed. They were thin-walled, 2 to 6 cells and irregular in shape and size compared with those of other $T$. mentagrophytes varieties (Fig. 3a). There were abundant pear-shape or more elongated microconidia. They were in a right-angle arrangement and along the sides of the mycelium (Fig. 3b). Many intermediate forms between microand macroconidia were also noted, while spiral bodies were few. The hair perforation test was positive at room temperature for 3 weeks (Fig. 4). The urease test on Christensen's urea agar slant (Eiken, Tokyo, Japan) was positive at $25^{\circ} \mathrm{C}$ for 2 days. The maximum growth temperature was $40^{\circ} \mathrm{C}$. Cultures of scale and hair of the barking squirrel and hedgehogs living together with the present animal were negative.

Mating tests: On salt-added 1/10-diluted Sabouraud's agar medium (glucose $0.2 \%$, Neopeptone Difco, $0.1 \%, \mathrm{MgSO}_{4} \cdot 7 \mathrm{H}_{2} \mathrm{O} 0.1 \%$, $\mathrm{KH}_{2} \mathrm{PO}_{4} 0.1 \%$, Bacto agar $2 \%$ ), the mating test was conducted with both plus $(+)$ and minus $(-)$ mating types of $A$. simii, $A$. vanbreuseghemii and African and Americano-European races of $A$. benhamiae (Table 1). Many gymnothecia with ascospore formation were produced between the African race (-) strain of $A$. benhamiae and the animal isolate. Most ascospores picked out of the inside of a gymnothecium under a stereoscope

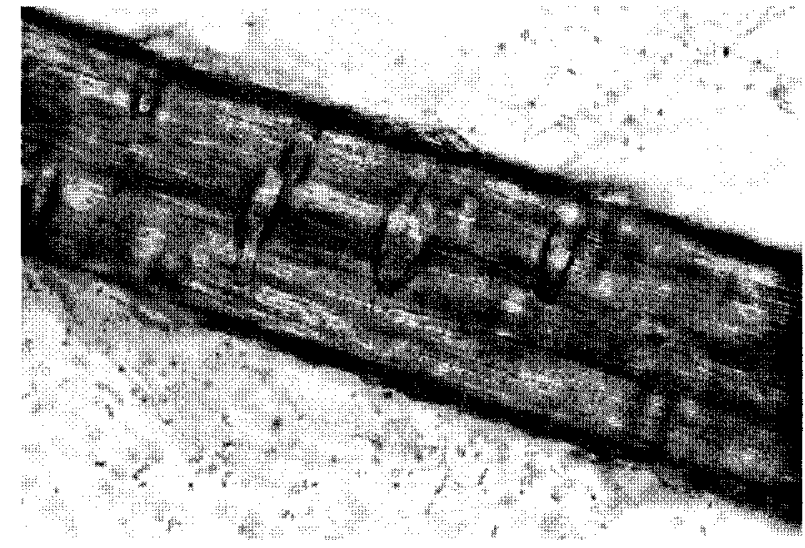

Fig. 4 Positive in the hair perforation test at room temperature for 3 weeks (lactophenol cotton blue staining, $\times 400$ ).

and suspended in sterilized water germinated on PDA at $25^{\circ} \mathrm{C}$ after 1 to 2 days. Sexual activity was also stimulated in the mating between the Americano-European race $(-)$ strain of $A$. benhamiae and the (-) strain of $A$. simii but ascospores between those pairs were not detected. Molecular biological identification of $T$. mentagrophytes var. erinacei: DNA was extracted from the culture on PDA at $25^{\circ} \mathrm{C}$ for 14 days by the benzyl chloride method ${ }^{8)}$, and the internal transcribed spacer region including 5.8S of ribosomal RNA gene (ITS1-5.8S-ITS2 rDNA) was detected by PCR with the primer set of ITS5 (5'-GGA AGT AAA AGT CGT AAC AAG G-3') and ITS4 (5'-TCC TCC GCT TAT TGA TAT GC-3'). The PCR product was labeled by ITS1 (5'-TCC GTA GGT GAA CCT GCG G-3') and ITS4. The PCR product was directly sequenced in both strands using a DNA sequencing kit BigDye $^{\mathrm{TM}}$ Terminator Cycle 
Table 1. Tester strains used for mating tests

\begin{tabular}{|c|c|c|}
\hline \multicolumn{2}{|c|}{ Tester strains } & \multirow[t]{2}{*}{$\begin{array}{l}\text { Mating behavior between } \\
\text { the hedgehog isolate }\end{array}$} \\
\hline A. simii & & \\
\hline mating type + & IFM49897 $=$ CBS448.65 & infertile \\
\hline mating type - & IFM $49898=\mathrm{CBS} 417.65$ & stimulated \\
\hline \multicolumn{3}{|l|}{ A. vanbreuseghemii } \\
\hline mating type + & IFM41148=RV27960 & infertile \\
\hline mating type - & IFM41149=RV27961 & infertile \\
\hline \multicolumn{3}{|c|}{ A. benhamiae Americano-European race } \\
\hline mating type + & IFM48142=RV26678 & infertile \\
\hline mating type - & IFM48143=RV26680 & stimulated \\
\hline \multicolumn{3}{|c|}{ A. benhamiae African race } \\
\hline mating type + & IFM48144=RV30000 & infertile \\
\hline mating type - & IFM48145=RV30001 & fertile \\
\hline
\end{tabular}

IFM: Research Center for Pathogenic Fungi \& Microbial Toxicoses, Chiba University, Chiba CBS: Centraalbureau voor Schimmelcultures, Baarn, The Netherlands RV: Institute de Medicine Tropicale, Antwerp, Belgium

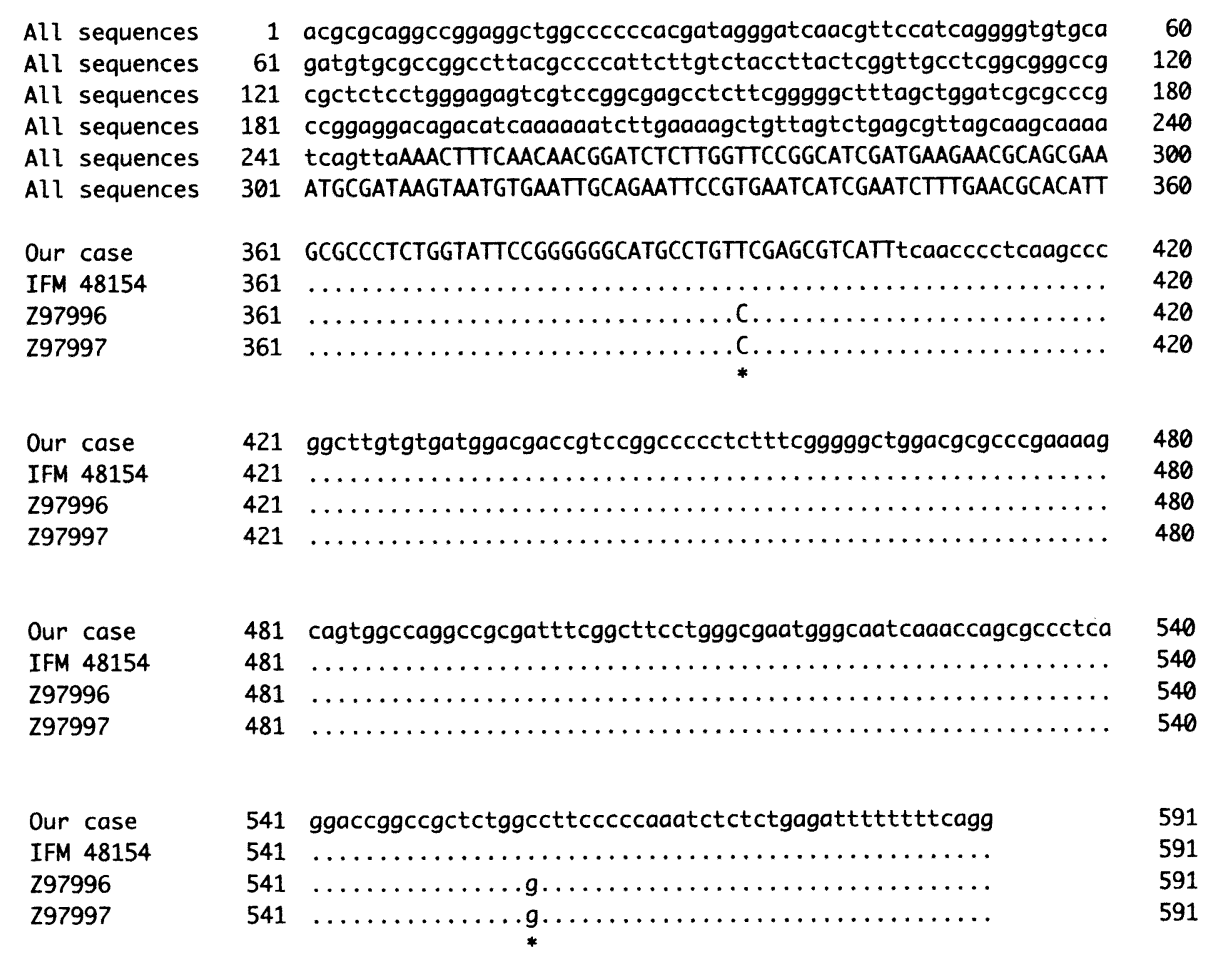

Fig. 5 Comparison of internal transcribed spacer (ITS) 1-5.8S-ITS2 ribosomal RNA gene.

*; Indicates a different base. All sequences were identical from the first to 360th base.

Lowercase letters; ITS regions. Uppercase letters; $5.8 \mathrm{~S}$ region.

Sequencing Ready Reaction, ABI PRISM ${ }^{\circledR}$ Applied Biosystems, Tokyo), and sequenced by a sequencer (ABI PRISM ${ }^{\circledR} 3100$ Genetic Analyzer, AB Applied Biosystems, Hitachi). The nucleotide sequence data were analyzed with genetic information processing software, GENETYXMAC Ver. 11.2.1 (Software Development, Shibuya, Tokyo), and a total of 591 bases determined. Homology search of the sequences was conducted by BLAST of the National Center for
Biotechnology Information (NCBI, Bethesda, MD, USA), and $99.7 \%$ homology was revealed with the partial sequence of ITS1-5.8S-ITS2 rRNA gene of $T$. mentagrophytes var. erinacei in accession numbers Z97996 and Z97997. In addition, $100 \%$ homology was confirmed with $T$. mentagrophytes var. erinacei (IFM 48154=RV28924, DDBJ accession number AB078899) which was kept at the research center. The sequence of the present isolate was submitted to DDBJ with the 
accession number AB078898 (Fig. 5).

Based on the results of morphological and physiological studies, mating tests, and homology search of the partial sequence of the ITS region for the hedgehog isolate, the teleomorph was identified as $A$. benhamiae, and the anamorph as T. mentagrophytes var. erinacei.

Treatment and prognosis: Oral administration of ivermectine against the mites at a dose of 300 $\mu \mathrm{g} / \mathrm{kg}$ per week was started from the first day of the visit. Although the mites disappeared on the 18th day of treatment, the administration was continued for 4 weeks. The fungus grew on the agar medium, and then antifungal therapy was started from the 12th day of the clinical history. Griseofulvin at a dose of $50 \mathrm{mg} / \mathrm{kg}$ per day was given orally for 6 weeks. Fungal culture by a hairbrush method and direct culture of the quills became negative after 4 weeks of treatment. There has been no relapse to date.

\section{Discussion}

In 1960, Marples and Smith first reported the isolates of $T$. mentagrophytes from hedgehogs and persons who contacted the animals in New Zealand. According to their description, their morphological and physiological characteristics were significantly different from those of the other varieties of $T$. mentagrophytes. The following points of difference have been given: elongated microconidia formed along sides of the mycelium, abundant intermediate forms between micro- and macroconidia, few spiral bodies and a diffusible brilliant lemon-yellow pigment on the reverse. Therefore, Smith and Marples proposed a new variety of $T$. mentagrophytes var. erinacei for the isolates $^{9,10)}$.

Since then, many reports on dermatophytoses attributable to the organism in humans and hedgehogs have been made. Although the hedgehogs are classified into 14 species of 4 genera ${ }^{7)}$, $T$. mentagrophytes var. erinacei has been isolated only from Western European hedgehogs (Erinaceus europaeus) of the genus Erinaceus. The natural habitats of the animal species are the United Kingdom and Northern and Western Europe, and the species immigrated into New Zealand with the human immigration. The original home of the four-toed hedgehog (Atelerix albiventris) is from Senegal to the Zambezi River basin. It is smaller than the Western European hedgehog, has a white abdomen, and is characterized by lacking the first toe of the hind leg. The animal had previously been classified in the genus Erinaceus $^{11)}$ like Western European hedgehogs, but recently it has been classified as part of the genus
Atelerix $^{7)}$. The four-toed hedgehog has spread all over the world as a pet animal, most of them are domesticated with wild populations reported to be few. It is unknown whether the present animal was imported directly from Africa or grown in Japan, but the animal had skin lesions at the time of purchase, and it is suggested that the causative fungus in the present case might have been transmitted from a wild individual.

Some isolates of a dermatophyte species reportedly were obtained from wild four-toed hedgehogs in Nairobi, Kenya in $1972^{12)}$. The isolates were morphologically similar to $T$. mentagrophytes var. erinacei. However, they were different in the physiological characteristics of urease activity, growth rate and having medium coloration. Since they mated with the teleomorphic species $A$. benhamiae, Collinge et al. concluded that they were conspecific ${ }^{13)}$.

The present isolate was very similar to the Kenyan isolates morphologically and physiologically. In addition, the homology in ITS1-5.8S-ITS2 rRNA gene with $T$. mentagrophytes var. erinacei was more than $99.7 \%$. Therefore, it is possible that the Kenyan isolates are $T$. mentagrophytes var. erinacei in anamorph. Takashio ${ }^{14)}$ observed that the Kenyan isolates produced mature gymnothecia in mating with the $A$. benhamiae African race but immature ones with the Americano-European race of the species, and placed them in a new variety $A$. benhamiae var. erinacei. Interestingly, the mating type of Kenyan isolates was $(-)$, although our isolate had $(+)$ mating type.

According to Makimura ${ }^{15)}$, the sequences of ITS1 rRNA gene were sufficient for species identification of dermatophytes. As T. mentagrophytes var. erinacei, all the strains registered at the DDBJ/EMBL/GenBank (accession Nos. Z97996, Z97997 and AB011455) showed 100\% homology in a comparison of the ITS1 region by BLAST analysis (GenBank). The present isolate also showed 100\% homology. However, it did show a 5-base difference from $A$. benhamiae African race (AB048193) and a 6-base difference from $A$. benhamiae Americano-European race (AF 170457) in this region.

Further information on the ITS1-5.8S-ITS2 rRNA gene sequences revealed 100\% homology with T. mentagrophytes var. erinacei (AB078899) and $99.7 \%$ homology with Nos. Z97996 and Z97997 by a 2-base difference. The hedgehog strain, in contrast, showed a 9-base difference (98.4\% homology) from the A. benhamiae African race (AB048193) and a 12-base difference (98.0\%) from the A. benhamiae Americano- 
European race (AF170457). Furthermore, the homology of ITS1-5.8S-ITS2 rRNA gene sequences between $A$. vanbreuseghemii (AF170452) and the present isolate was at the rate of $89.4 \%$ and that between $A$. simii (AF170454) was $91.5 \%$.

From this homology search, the anamorph of the present hedgehog isolate is unquestionably $T$. mentagrophytes var. erinacei. Although the mating behavior of the isolate indicates that its teleomorph is the $A$. benhamiae African race, the fertility between F1 progeny is not yet clarified. Furthermore, the ITS1-5.8S-ITS2 rRNA gene is different from that of $A$. benhamiae African race. It is impossible to conclude that teleomorph of $T$. mentagrophytes var. erinacei is $A$. benhamiae African race from what we know now. These may suggest that the teleomorph of $T$. mentagrophytes var. erinacei is a close but different group peculiar to the anamorph.

In recent years, reports on imported mycoses such as coccidioidomycosis, paracoccidioidomycosis, and histoplasmosis have increased in Japan ${ }^{16)}$. These conditions have appeared in Japanese returning from endemic areas or in foreigners staying in Japan, but there has been no personto-person transmission. Therefore, the infections are very unlikely to spread widely in Japan, and if imported mycosis is suspected, a presumed diagnosis is possible from a patient's history of going abroad. For imported mycosis in animals, however, it is difficult to know their life history, whether wild, bred in Japan or abroad, and it is almost impossible to distinguish whether the disease is an imported mycosis. Additionally, such small animals are often kept in the home, and have occasions of close contact with the owner and family. T. mentagrophytes var. erinacei is known to cause zoonosis, and may cause mass infection within a family.

English and her colleagues ${ }^{17,18)}$ say that there are two routes of $T$. mentagrophytes var. erinacei infection for humans. One is a direct infection from Western European hedgehogs, and there have been several cases of mass infection within a family. The other is an indirect infection, and T. mentagrophytes var. erinacei adhering to the nests of Western European hedgehogs is reported to have survived for approximately one year under dry conditions in a laboratory. It is suggested that infected nest material can be an indirect infection source between humans and Western European hedgehogs. Although no infection to the owner's family in our case was observed, hedgehogs in Japan may also cause zoonosis due to direct and indirect infections.
The relationship with mite infections is significant. In an investigation in New Zealand, Smith and Marples ${ }^{10)}$ reported that infections with mites and $T$. mentagrophytes var. erinacei were observed simultaneously in $47.5 \%$ of wild Western European hedgehogs, and mites may become carriers to spread the dermatophyte infection. In our case, the parasitism of many mites was observed, and it also indicated a relationship with the dermatomycosis.

In New Zealand, T. mentagrophytes var. erinacei was reported to have been found in approximately $52.5 \%$ of wild Western European hedgehogs that were bred after immigration ${ }^{10}$. In Japan, it has been confirmed that Western European hedgehogs have become wild in Tochigi, Kanagawa, and Nara since $1987^{19}$. The four-toed hedgehogs can also become wild if they adapt themselves to the environment, and $T$. mentagrophytes var. erinacei could then spread to the natural world.

As stated, imported small animals such as hedgehogs and other rare rodents have recently become popular pets in Japan. In any case of an atypical $T$. mentagrophytes isolated from humans, we should from now on consider $T$. mentagrophytes var. erinacei.

\section{Acknowledgements}

This study was performed as part of the program "Frontier Studies and International Networking of Genetic Resources in Pathogenic Fungi and Actinomycetes" through Special Coordination Funds for Promoting Science and Technology from the Ministry of Education, Culture, Sports, Science and Technology of the Japanese Government in 2001. The research was partially supported by the Japanese Ministry of Health, Labor and Welfare through grant No. 12102301. We are very grateful to Drs. Hiroshi Ishizaki and Masako Kawasaki, Department of Dermatology, Kanazawa Medical University, Ishikawa, Japan for providing the tester strains of A. benhamiae.

\section{References}

1) Nakamura $Y$, Watanabe $\mathrm{S}$, Hasegawa $\mathrm{A}$ : Dermatomycosis in human and animals. Jpn J Med Mycol 40: 9-14, 1999.

2) Hata $Y$, Amagai M, Naka W, Harada R, Nishikawa T: Two cases of Trichophyton mentagrophytes infection contracted from a hamster and a chinchilla. Jpn J Med Mycol 41: 269-273, 2000.

3) Iizuka $T$, Ninomiya $J$, Hamaguchi $T$, Nagase M, Takiuchi I: Two cases of tinea due to Trichophyton mentagrophytes infection by a rabbit. 
Jpn J Med Mycol 38: 247-252, 1997.

4) Mizuno T, Kanaya T, Koyama K, Ito T, Tamura Y, Ito Y: Four cases of tinea due to Trichophyton mentagrophytes infection by a rat. Rinsho derma 20: 49-53, 1978 (in Japanese).

5) Kano R, Nakamura Y, Yasuda K, Watari T, Watanabe S, Takahashi $\mathrm{H}$, Tsujimoto $\mathrm{H}$, Hasegawa A: The first isolation of Arthroderma benhamiae in Japan. Microbial Immunol 42: 575578, 1999.

6) Kawasaki $M$, Aso $M$, Inoue $T$, Ohsawa $T$, Ishizaki $\mathrm{H}$ : Two cases of tinea corporis by infection from a rabbit with Arthroderma benhamiae. Jpn J Med Mycol 41: 263-267, 2000.

7) Wilson DE, Reeder DAM: Mammal Species of the World, P76-77, Smithsonian Institution Press, New York, 1993.

8) Zhu H, Qu F, Zhu LH: Isolation of genomic DNAs from plants, fungi, and bacteria using benzil chloride. Nucleic Acids Res 21: 52795280, 1993.

9) Marples MJ, Smith JMB: The hedgehog as a source of human ringworm. Nature 188: 867868, 1960.

10) Smith JMB, Marples MJ: Trichophyton mentagrophytes var. erinacei. Sabouraudia 3: 1-10, 1963.

11) Macdonald DW: The encyclopaedia of animals (Japanese edition). Vol. 6: p.18-25, Heibonsha, Tokyo, 1986.

12) Gregory MW, English MP: Arthroderma benhamiae infection in the central African hedgehog, Erinaceus albiventris, and a report of a human case. Mycopathologia 55: 143-147, 1975.

13) Collinge CA, Stockdale PM, Gregory MW: A mycological study of Arthroderma benhamiae from the central African hedgehog. Sabouraudia 12: 227-232, 1971.

14) Takashio M: The perfect states of dermatophytes and the reclassification of Trichophyton mentagrophytes on the basis of its perfect states. Nishinihon J Dermatol 38: 703-728, 1976 (in Japanese with abstract in English).

15) Makimura K: Species identification system for dermatophytes based on the DNA sequences of nuclear ribosomal Internal Transcribed Spacer 1. Jpn J Med Mycol 42: 61-67, 2001.

16) Fujio J, Nishimura K, Miyaji M: Epidemiological survey of the imported mycoses in Japan. Jpn J Med Mycol 40: 103-109, 1999.

17) English MP, Evans CD, Hewitt M, Warin RP: Hedgehog ringworm. British Medical J 1: 149151, 1962.

18) English P, Morris P: Trichophyton mentagrophytes var. erinacei in hedgehog nests. Sabouraudia 7: 118-121, 1969.

19) Ikeda $T$ : Distribution and problems in immigrant mammals in Japan. (Immigrant animals as environmental problems). Transactions of the Department of Literature, Hokkaido University 46: 195-215, 1997 (In Japanese). 